\title{
Effect of plant growth regulators on callus induction from leaf and petiole explants of Hummeid (Rumex vesicarius)
}

\author{
W.P.K.K. Wickramanayake ${ }^{a}$, Talaat Abdel-Fattah Ahmed ${ }^{a}$, Mohammed H. Alsafran ${ }^{b}$ \\ a Environmental Science Center, Qatar University \\ ${ }^{b}$ Agricultural Research Station, Qatar University
}

\section{Overview}

- Advancement in plant cell culture technology has permitted the increasing interest in harvesting plant metabolites from cell cultures.

- This technique is based on in vitro propagation of plant callus which contains many beneficial compounds under sterile conditions as the plant source or raw material for natural product extraction.

- In vitro production of plant sources acknowledges; conservation of plant species in natural habitats which has been a global issue due to over harvesting of medicinal plants for the natural products manufacturing and production of phytochemicals independent from seasonal and environmental limitations.

- Establishment of cell cultures is challenging. The main aim is to establish cell cultures as the starting material for the phytochemical analysis in Rumex vesicarius.

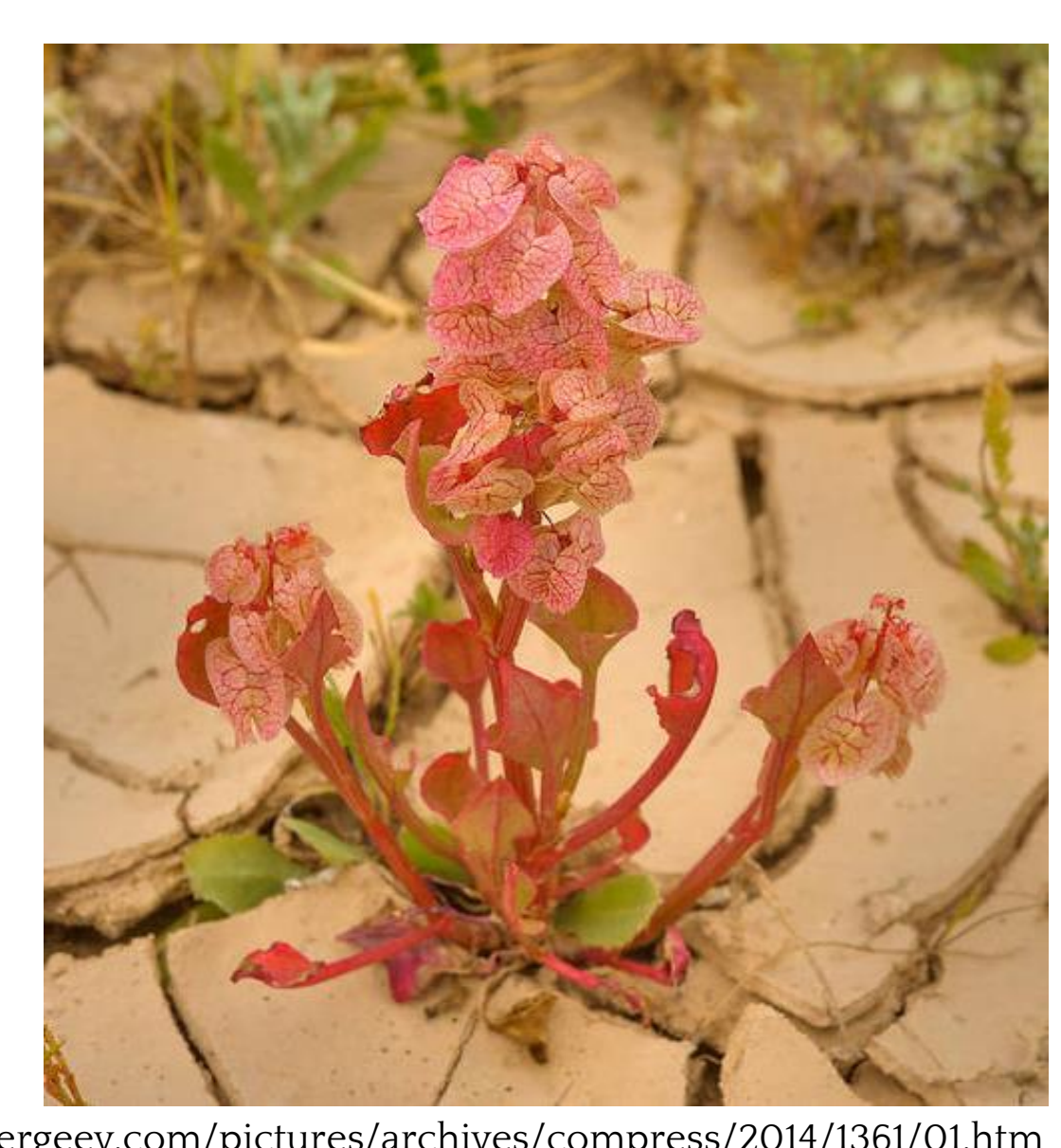

- Rumex vesicarius commonly known as Badder Dock, Hummeid (in Qatar). Its is a perennial flowering plant belongs to family Polygonaceae. This plant is documented as a native herb to tropical and temperate Asia, Africa, and introduced to Australia.

\section{Significance of Rumex vesicarius}

- An edible herb

- Medicinal uses: Aperient, astringent, diuretic and cooling. The roasted seeds can be used in the treatment of dysentery, the juice of the plant can be used in treating stomach problems such as bloating, nausea indigestion, alleviate the pain of toothache, good herbal remedy for jaundice, Dysentery and Hepatitis.

- Contains fairly high levels of oxalic acid.

\section{Acknowledgement}

This material is based upon work supported by M-QJRC-2O2O-10 project funds and ESC laboratory facilities.

\section{Methodology}

\section{Establishment of in vitro mother plant} stock to obtain explants

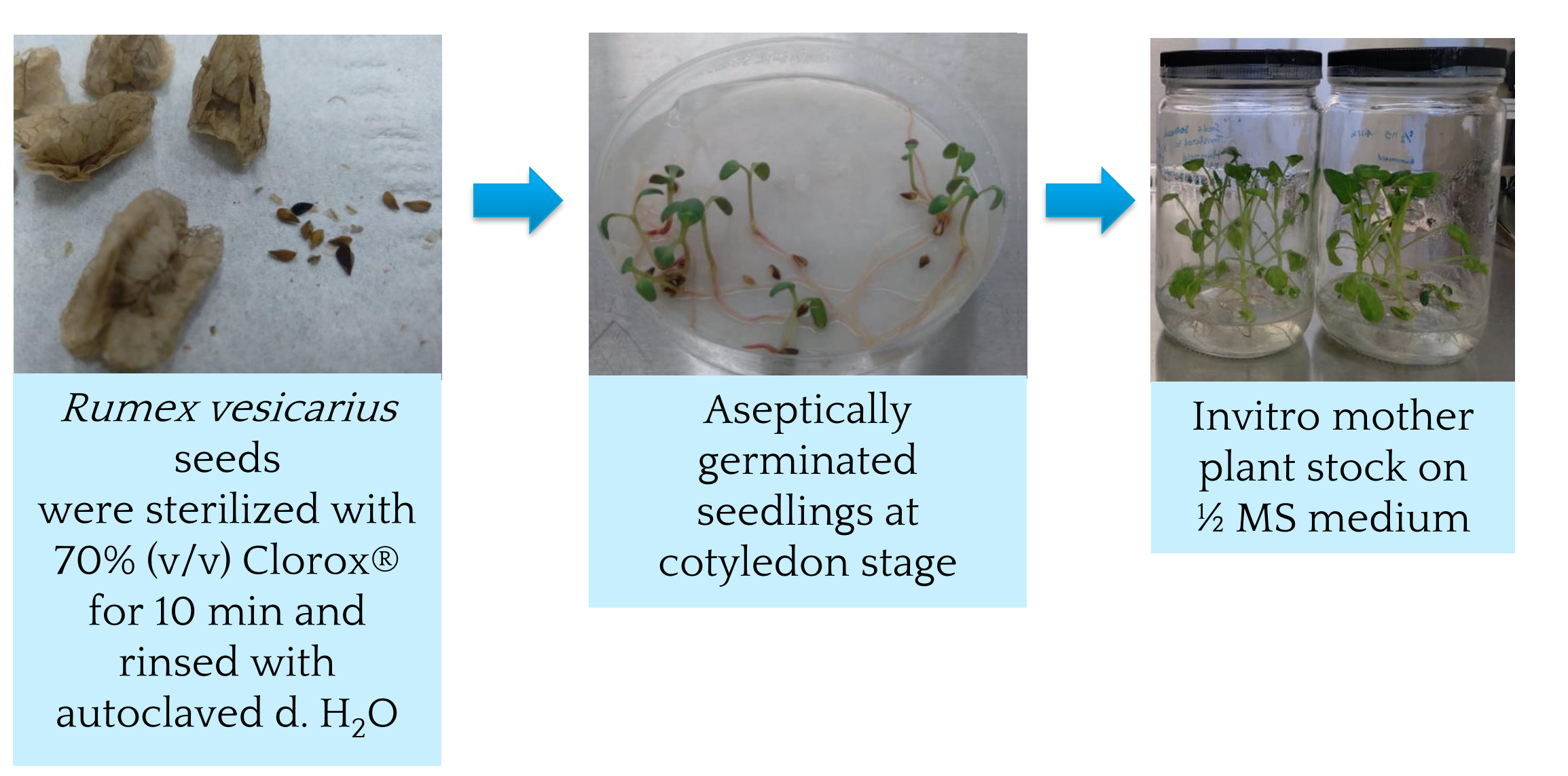

\section{Preliminary study}

Different types of explants acquired from different Days After Sawing (DAS) seedlings were initiated in same basal medium (MS) with different supplements and Plant Growth Regulators (PGR).

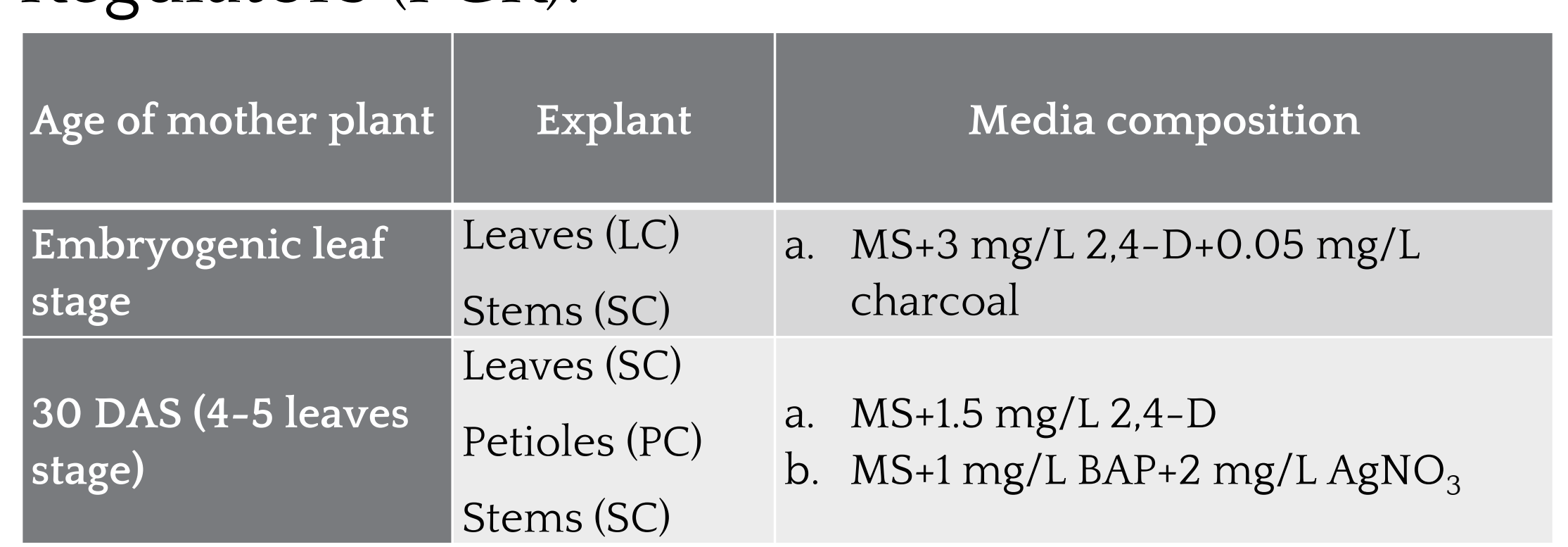

\section{Optimization of Rumex vesicarius callus} induction

\begin{tabular}{|c|c|c|c|c|}
\hline \multicolumn{2}{|c|}{ Auxin } & \multicolumn{2}{|c|}{ Cytokinin } & Auxin + Cytokinin \\
\hline $\begin{array}{l}\mathrm{MS}+2 \mathrm{mg} / \mathrm{L} \\
2,4-\mathrm{D}\end{array}$ & $\begin{array}{l}\mathrm{MS}+2 \mathrm{mg} / \mathrm{L} \\
\mathrm{IBA}\end{array}$ & $\begin{array}{c}\mathrm{MS}+2 \mathrm{mg} / \mathrm{L} \\
\mathrm{BAP}\end{array}$ & $\begin{array}{l}\mathrm{MS}+2 \mathrm{mg} / \mathrm{L} \\
\text { Kinetin }\end{array}$ & $\begin{array}{cc}\mathrm{MS}+1 \mathrm{mg} / \mathrm{L} & \mathrm{MS}+2 \\
2,4-\mathrm{D} & \mathrm{mg} / \mathrm{L} \text { BAF }\end{array}$ \\
\hline
\end{tabular}

Two different types of explants acquired from 30 DAS seedlings were initiated on same basal media with different PGR.

\section{Evaluation of Callus formation}

-Increase of biomass

-Callus formation frequency (\%) (The number of explants forming callus was counted and callus formation frequency was recorded as a percent of the 7 explants replicates)

-Morphology (colour and texture) and degree of callus formation (1-4)

One-way ANOVA was used to determine statistically significant differences between the means of biomass increase under each treatment and each treatment means were compared by Tukey's HSD at 0.05 confidence level.
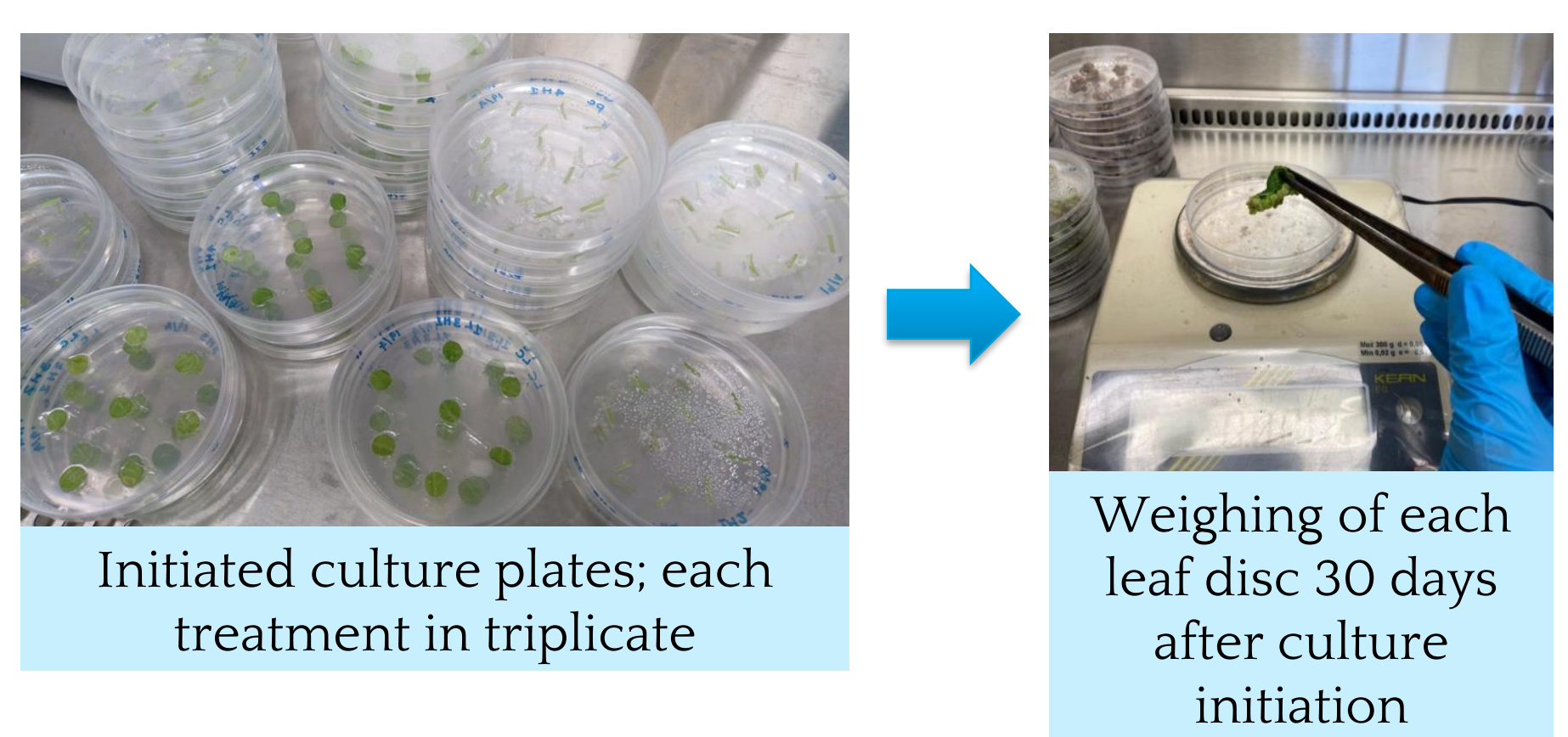

\section{Results}

\section{Preliminary study}

- The cultures initiated with the explants obtained from embryogenic leaf stage on the basal MS media supplemented with 3 $\mathrm{mg} / \mathrm{L} 2,4-\mathrm{D}$ and $0.05 \mathrm{mg} / \mathrm{L}$ Charcoal; both leaf and stem explants started to form cell masses which were gradually turned into brown and dead after 30 days.

- Callus formation was not observed in SC, but successful shoot generation was observed which were recovered and initiated in 1/2 MS to generate new plants.

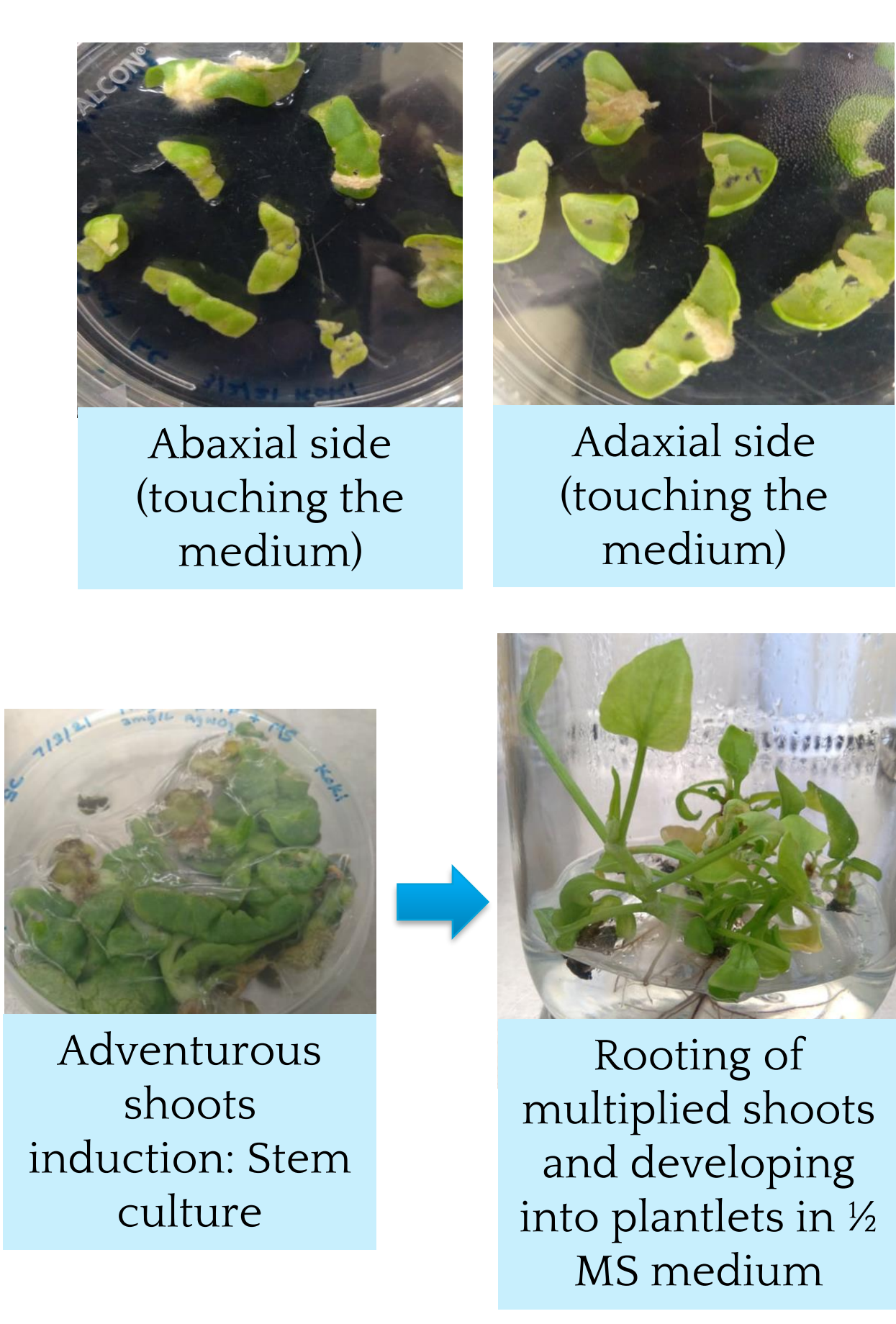

- Some leaf explants produced white friable callus while some leaf explants initially resulted hairy structures which were gradually turned into roots in the respective media.

- Basically callus formation initiated from the main veins and midrib. The explants placed abaxial side up resulted comparatively higher rate of callus formation

\section{Optimization of Rumex vesicarius callus induction}
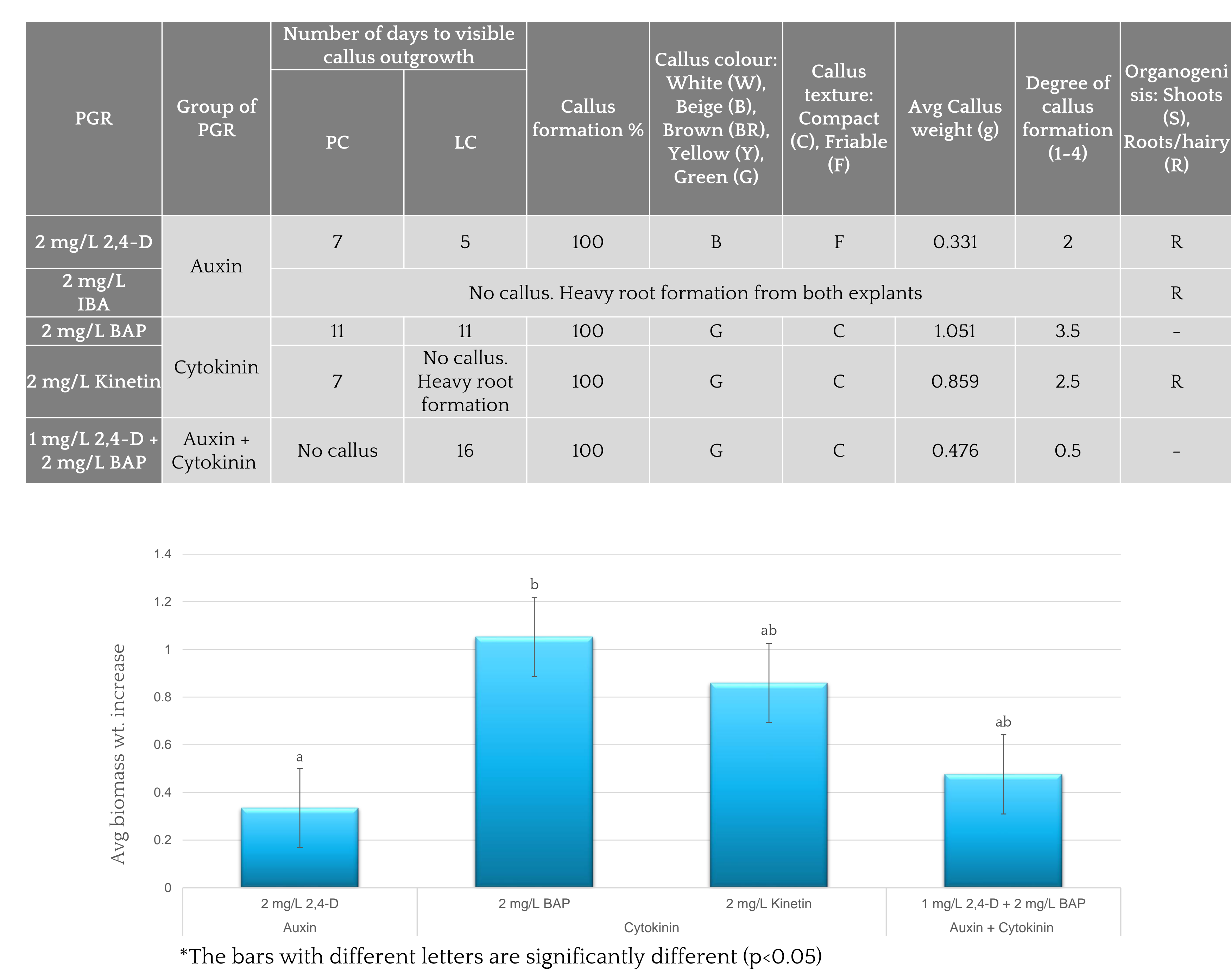

- Culture conditions: Photoperiod 5 hrs, Relative humidity 30\%, temperature 26 ${ }^{\circ} \mathrm{C}$, culture period 30 days.

- Initiation of in vitro cultures is $100 \%$ in all culture media.

- The highest biomass increase is obtained on the MS medium supplemented with 2mg/L BAP.

- Callus texture and colour is influenced by different explant and PGR

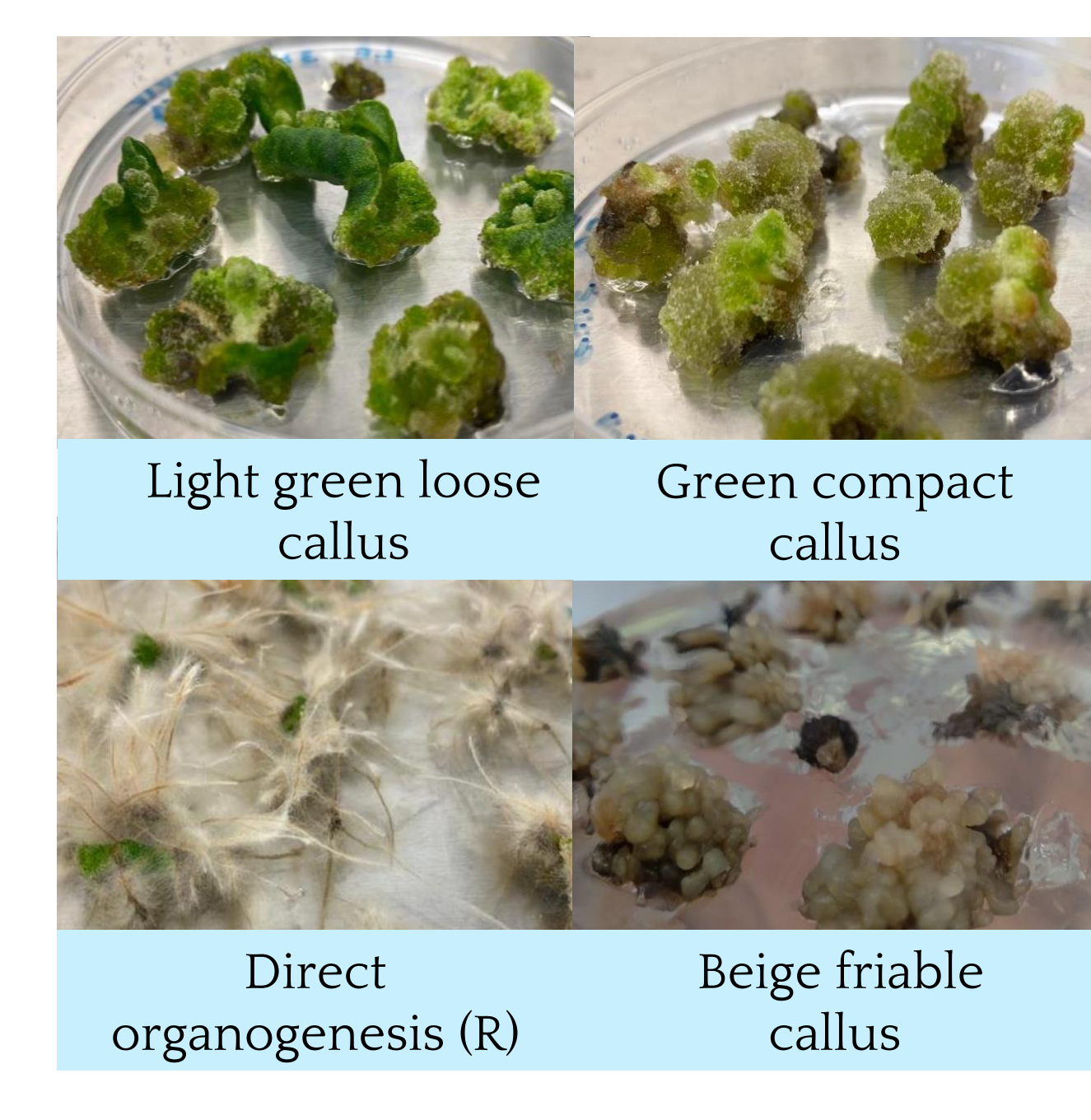

\section{Conclusion}

- An efficient plant tissue culture protocol has optimized to initiate Rumex vesicarius callus cultures in a relative short time and high multiplication rate.

- Accordingly, leaf explants acquired from 30 DAS seedlings initiated in MS medium supplemented with 2mg/L BAP resulted the highest callus biomass production.

- However, the petiole and leaf cultures on MS+2mg/L 2,4-D medium resulted completely different textured and colour callus with high multiplication and survival rate.

- Explants obtained at embryogenic leaf stage is not suitable for the callus induction of Rumex vesicarius plants. Furthermore, there was no browning effect/oxidation, hence charcoal supplement in the media can be omitted which leads absorption of the PGRs in the media and can half the $3 \mathrm{mg} / \mathrm{L} 2,4-\mathrm{D}$ in the media.

- The knowledge of rooting media would be helpful to establish organ (root) cultures upon the evidence of presence of the bioactive compounds in elevated concentrations in Rumex vesicarius roots. 